\section{Enlightenment - Cathodoluminescence: The Bright Technique, Part I}

Paul Wright, Oxford Instruments Ltd, Eynsham, UK

This is the first in a series of articles that serve to identify what scanning Cathodoluminescence can offer the materials technologist. Whilst begining with Electronic Materials, Geological,Ceramic and Diamond applications will be covered over the next installments concluding with a reveiw of instrumentation needed to perform the studies covered in the final chapter.

Cathodoluminescence $(\mathrm{CL})$ is the most important optical phenomenon used by all electron microscopists. It is the process by which electrons are converted into light in the Everhart Thornley secondary electron detector and also in the screens and cameras of TEMs.

Cathodoluminescence, however, can be used as an analysis technique in its own right to study materials which exhibit this phenomenon'. Amongst these are semiconductors of all types used for devices, optoelectronics and light emitting materials, geological materials, ceramics and diamond materials.

Cathodoluminescence arises as a result of electrons being excited into the conduction band of these materials, and subsequently recombining with holes left in the valence band. The energy difference is equalised by the emission of a photon. Depending on the process of recombination the photons will have the energy of the bandgap in the case of direct transitions or less than that in the case of indirect transitions.

\section{Semiconductors}

It is possible within this important class of materials to observe many of the defects that plague their successful manufacture. Gallium arsenide layers on silicon for use in integrated optoelectronic circuits are prone to cracking because of strain set up by the lattice mismatch between the silicon substrate and the GaAs epilayer (lattice mismatch @ 4\%). These cracks can hardly be seen in a secondary electron image as illustrated in Figure 1. However the reduction in strain which occurs near the edges and along the cracks alters the wavelength of $\mathrm{CL}$ emission as indicated in the spectra in Figure 2 which were from the points (a) and (b) in Figure 1.

This area of strain relaxation can be visualised by setting the monochromator to the wavelength that corresponds to the unstrained material. Figure 3 shows the unstrained regions which are emitting at $800 \mathrm{~nm}$ which corresponds to an energy transition of $1.549 \mathrm{eV}^{2}$

Other popular uses of $\mathrm{CL}$ are for the studies of quantum confined structures (wells, wires \& dots) which are being developed for diode laser structures. Here $\mathrm{CL}$ can assist in observing the uniformity of the emission at different wavelengths as shown in the examples as follows.

Here InGaAs quantum wells have been grown onto $v$-grooves on semi-insulating $\mathrm{InP}$. CL spectroscopy at room temperature indicated the presence of two major peaks as shown in Figure 4.

Setting the monochromator to these two emission wavelengths allows a pair of complimentary images to be taken which indicate not only the spatial distribution of the emission centres but also areas where the emission is degraded because of defects in the structures. Many of these effects are not visible by any other imaging technique and certainly not with the high spatial resolution obtained with $\mathrm{CL}$.

One of the most vibrant areas of opto electronics research at present is in the production materials that emit in the UV and blue regions of the electromagnetic spectrum.

Blue LED's and laser diodes have applications as diverse as diode based traffic signals with enormous power savings to using blue light to write increased volumes of data onto optical discs as a result of the shorter wavelength.

One of the many wide band gap materials that has been proposed for this purpose is gallium nitride.

Recent research by $\mathrm{CL}$ into gallium nitride based materials for blue LED's has allowed the sites for different emissions to be resolved. Previous photoluminescence (PL) studies have been incapable of providing this spatial information although have provided excellent spectral information as displayed in the upper two spectra of Fig 7 . CL has provided the spectral information in the lower spectrum and by imaging both the blue (364 nm) and yellow $(559 \mathrm{~nm})$ emissions it has been possible to observe the different sites responsible for these effects which are shown in Figures 9 and 10 .

Interestingly the light emission from the band edge and yellow regions in these undoped materials is inhomogeneous. The intensity of the band edge and yellow emissions correlate with columnar grain sizes obtained by TEM. It is hypothesised that the sources of the yellow emission are either dislocations at low angle grain boundaries in the material or point defects which nucleate at the dislocations ${ }^{3}$

Dislocations due to lattice mismatch can also be observed in epilayers of other materials. In Fig 10 a network of misfit dislocations are observed in a GaAsP epilayer on a GaP substrate at room temperature. These would be impossible to observe by other imaging techniques.

\section{Acknowledgements}

The author would like to thank Carl Norman, IRC for Semiconductor Studies at Imperial College, London; Fernando Ponce, Xerox Corporation, Palo Alto, USA and David Thompson, MacMaster University, Ontario, Canada for the important contributions made.

1 Yacobi, B.G., Cathodoluminescence Microscopy of Inorganic Solids, Plenum Press ISBN 0-306-43314-1.

2 Norman, C. and Murray, R, Microscopy of Semiconducting Materials, Oxford 1993.

3 Ponce, F.A. et al, Applied Physics Letters in press.

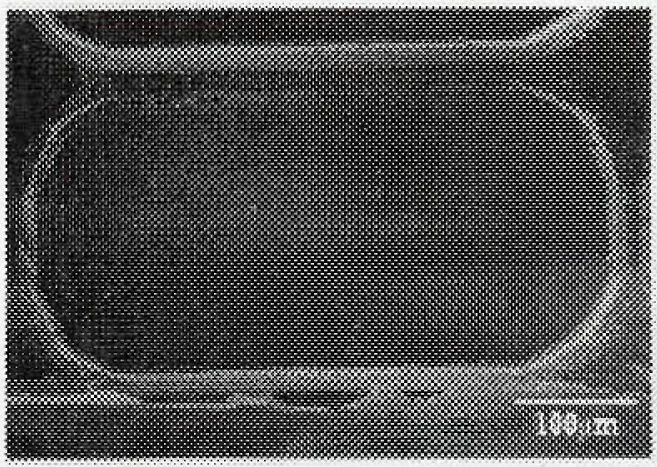

Figure 1: Secondary electron image of gallium arsenide layer on a patterned silicon substrate.
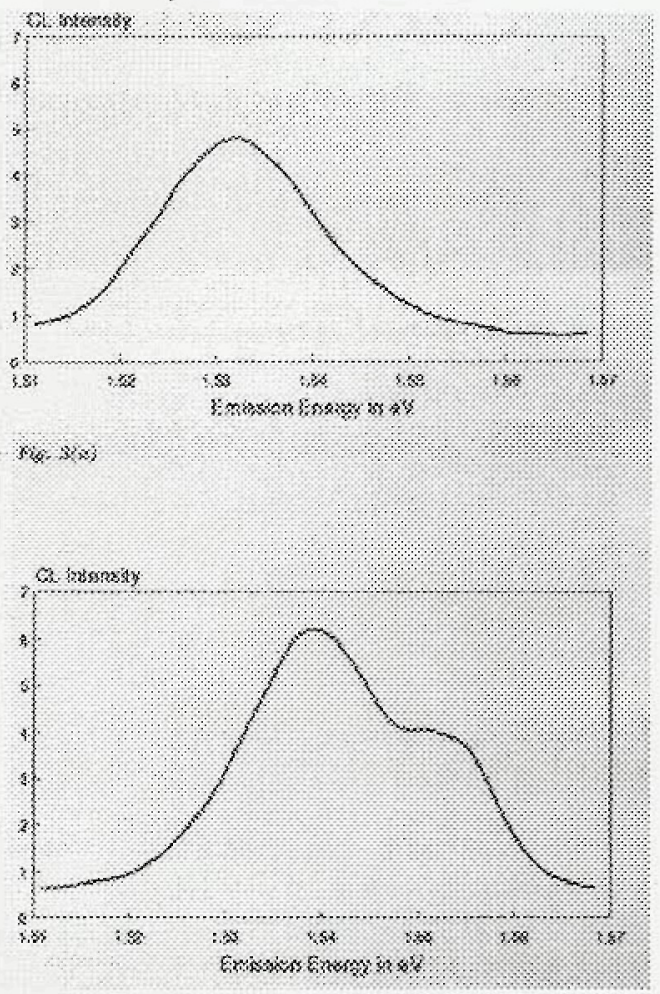

Figure 2: CL Spectra from points a \& b in Figure 1. 


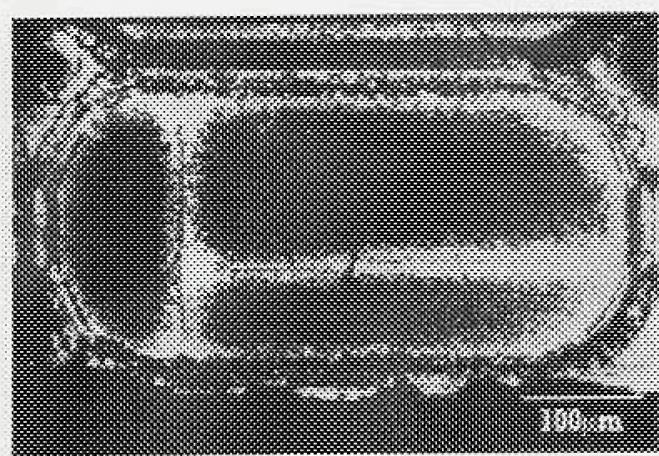

Figure 3. Monochromatic CL Image at $800 \mathrm{~nm}$ which corresponds to the area of reduced strain due to edge effects and microcracks.

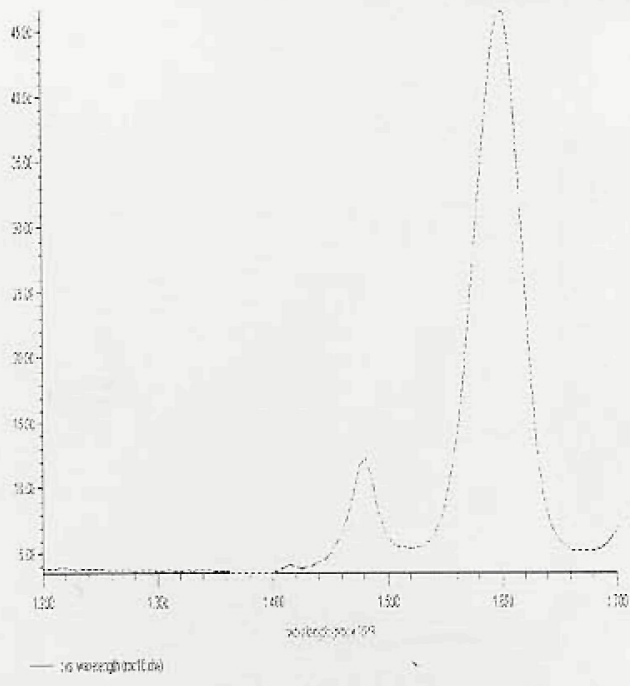

Figure 4: CL Spectra of InGaAs quantum well on $\ln P$ v-groove substrate

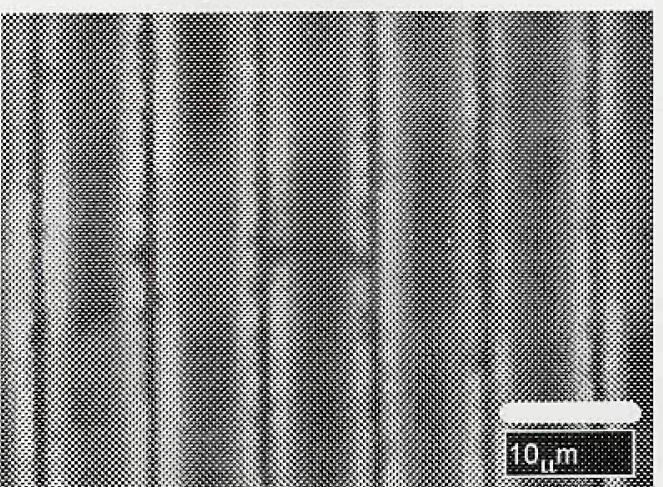

Figure 5: $\mathrm{CL}$ image at $1422 \mathrm{~nm}$ of InGaAs quantum well on InP v-groove substrate.

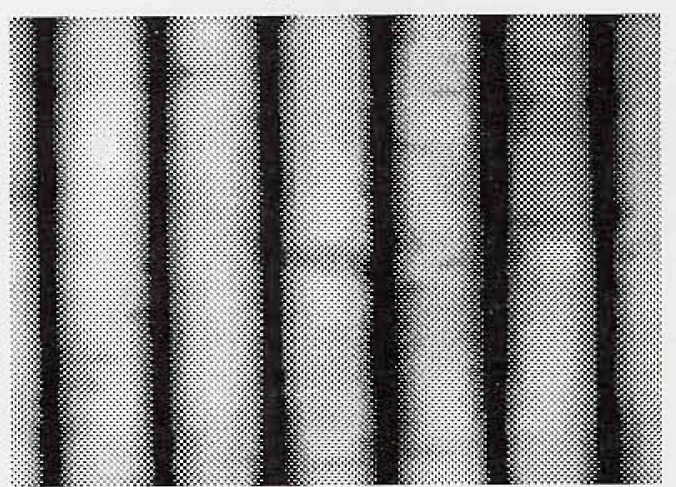

Figure 6: $\mathrm{CL}$ image at $1577 \mathrm{~nm}$ of $\mathrm{InGaAs}$ quantum well on InP v-groove substrate

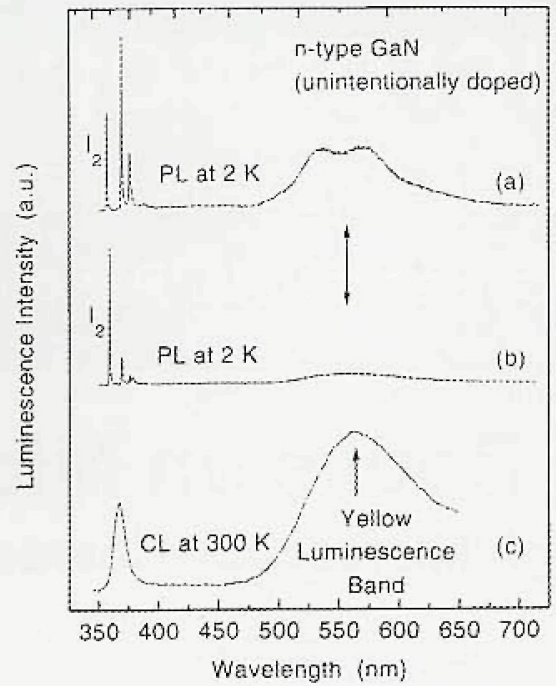

Figure 7: Luminescence spectra of non-intentionally doped GaN thin films.

(a) with uniform surface morphology

(b) with a surface dominated by hexagona crystallites

(c) $\mathrm{CL}$ at room temperature

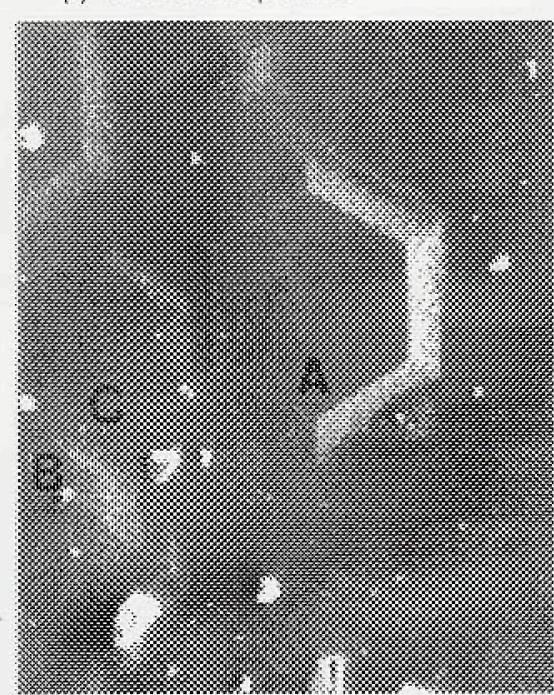

Figure 8: Secondary electron image of undoped GaN film showing the morphology

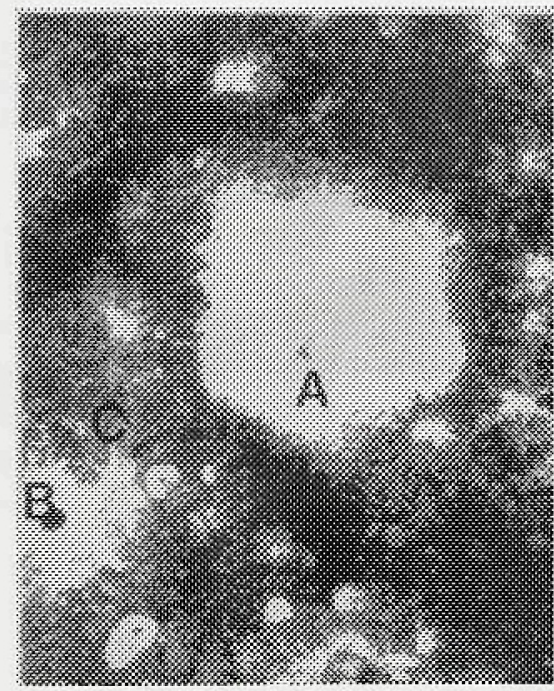

Figure 9: Monochromatic $\mathrm{CL}$ image at $364 \mathrm{~nm}$ showing that the band-to-band emissions are distributed only in the crystallites

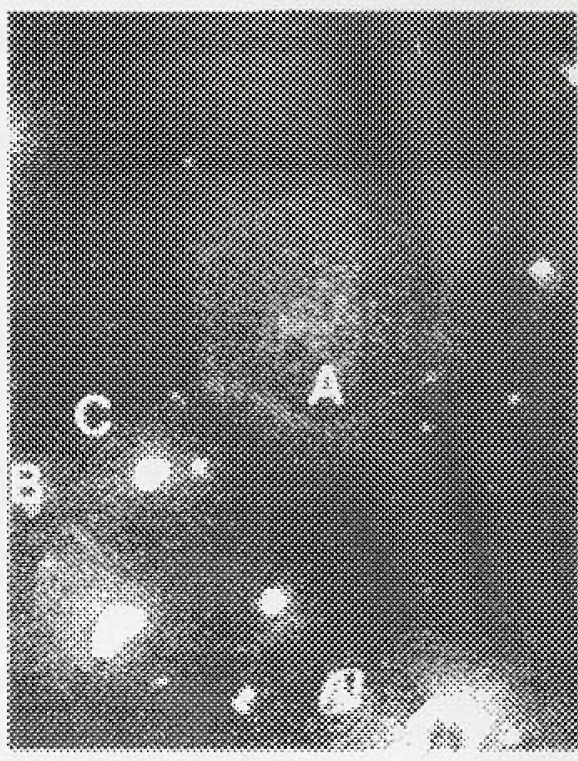

Figure 10: Monochromatic CL image at 559 am showing that the yellow emissions are distributed in the crystallites and in the boundaries

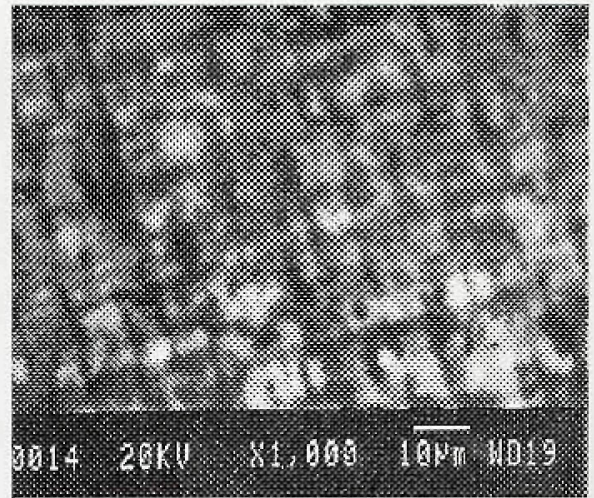

Figure 11. CL Image of a network of misfit dislocations in a GaAsP epilayer on a GaP substrate at room temperature

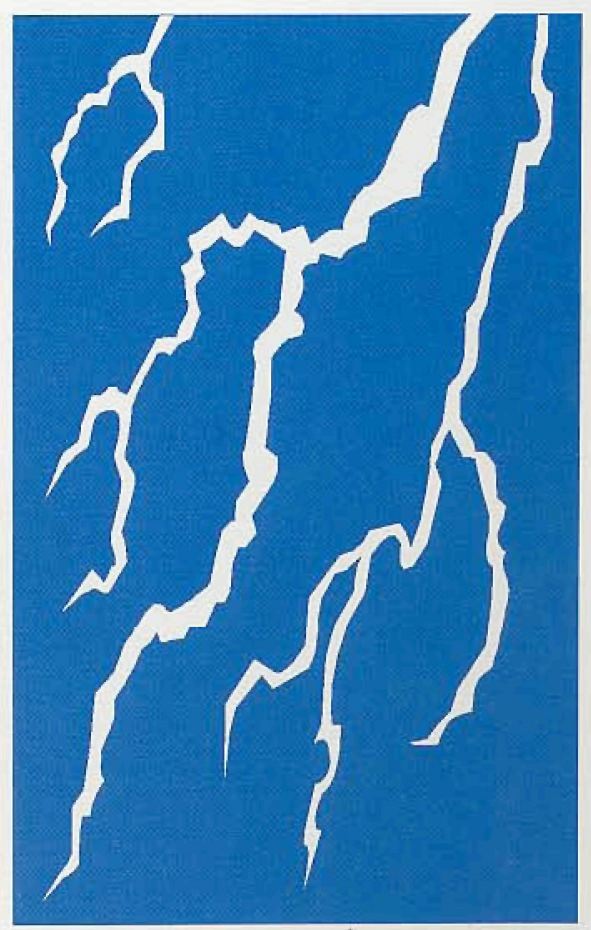




\section{Lehigh Microscopy Courses SEM, X-ray Analysis, AEM, AFM}

for Materials Engineers, Geologists, Biologists, Polymer Scientists

\section{Plenum Books on Microscopy by Lehigh Microscopy Course Authors}

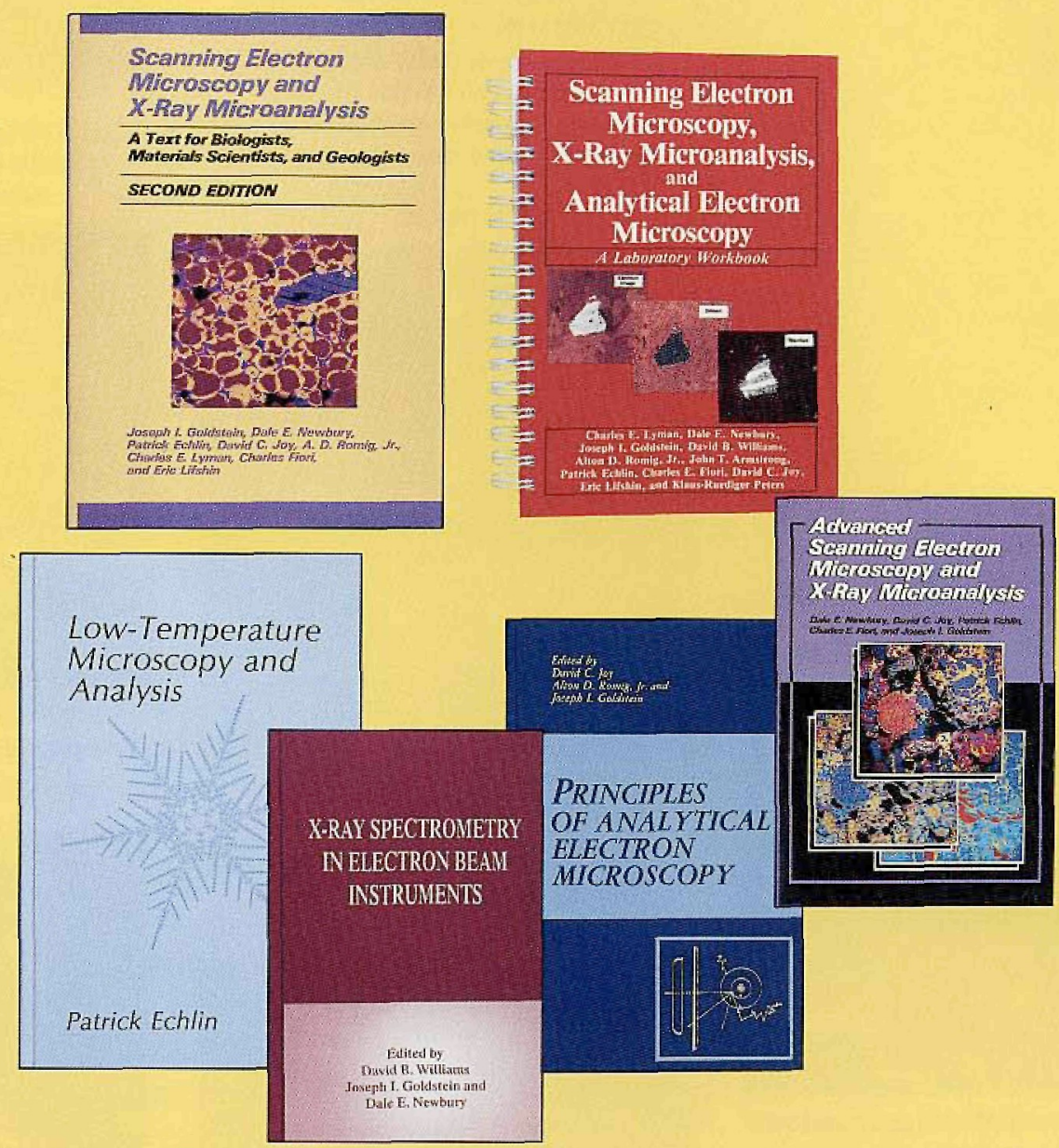
J. I. Goldstein et al., Scanning Electron Microscopy and X-ray Microanalysis, 2nd Edition..
$\underline{\text { US/Canada }}$
C. E. Lyman et al., SEM, X-ray Microanalysis, and AEM: A Laboratory Workbook.
$\$ 55.00$
D. E. Newbury, et al., Advanced Scanning Electron Microscopy and X-ray Microanalysis........... \$ \$49.50
D. C. Joy, et al., eds., Principles of Analytical Electron Microscopy.............................................. \$\$ \$55.00
D. B. Williams, et al., eds., X-ray Spectrometry in Electron Beam Instruments............................ \$ \$79.50
P. Echlin, Low-Temperature Microscopy and Analysis.
$\$ 95.00$

Forthcoming in 1996:

D. B. Williams and C. B. Carter, Transmission Electron Microscopy: A Textbook for Materials Science (Book prices are 20 percent higher outside the U.S. and Canada)

To order call: $\quad 1-800-221-9369$ or

write: $\quad$ Order Dept., Plenum Publishing, 233 Spring Street, New York, NY 10013-1578 


\section{Scanning Electron Microscopy and X-ray Microanalysis}

\section{The Lehigh Basic Course}

(June 10-14, 1996)

For scientists, technicians, engineers, and technical managers. June 1996 marks the 26th year of this course.

Topics include: Image formation, optimizing SEM parameters under operator control, high resolution $\mathrm{SEM}, \mathrm{x}$-ray detectors, qualitative and quantitative $\mathrm{x}$-ray analysis, image display and processing.

Materials topics include SEM of electronic materials, polymers and fibers, and fractography.

Biological topics include low temperature specimen preparation and immunocytochemical methods.

Instrumentation: 10 electron beam instruments with EDS, WDS, and digital imaging systems.

Textbooks Provided: Both texts were written by the course lecturers:

Scaming Electron Microscopy and X-ray Microanalysis, Plenum Press, 1992

SEM,X-ray Microanalysis, and AEM: A Laboratory Workbook, Plenum Press, 1990

\section{Advanced Scanning Electron Microscopy with Digital Image Processing}

(June 17-20, 1996)

Advanced treatment of high resolution SEM - low voltage SEM • environmental SEM • digital image acquisition - Monte Carlo simulations of electron beam-specimen interactions • electron detectors • quantitative stereo microscopy $\bullet$ stereology $\bullet$ image processing with personal computers

\section{Quantitative X-ray Microanalysis}

\section{of Bulk Specimens and Particles}

(June 17-20, 1996)

Advanced topics include: ZAF and $\phi(\rho z)$ calculations using personal computers - WDS and EDS detectors - quantitative analysis of thin particles and rough specimens • light element analysis • trace element analysis • strategies for applying microanalysis techniques • specimen preparation

\section{Analytical Electron Microscopy} and Quantitative Analysis of Thin Specimens

(June 17-20, 1996)

Advanced topics include: TEM/STEM and dedicated STEM instruments $\cdot$ Z-contrast $\cdot \mathrm{x}$-ray microanalysis • electron energy loss spectrometry $\bullet$ quantitative analysis $\cdot$ compositional imaging • convergent beam electron diffraction • microcomputer calculations • thin specimen preparation

\section{Atomic Force Microscopy}

\section{and Other Scanned Probe Microscopies}

(June 18-21, 1996)

Atomic force microscopy • scanning tunneling microscopy • scanning tunneling spectroscopy • feedback control • tip fabrication • tip-sample interactions • scan calibrations • in-situ imaging • UHV imaging • imaging in air and liquids $\bullet$ image processing $\bullet$ near-field optical probes $\cdot$ metrology lateral force microscopy • electrochemical STM/AFM • other emerging scanned probe techniques

For a detailed course brochure about these courses CONTACT:

Sharon Coe, Materials Science and Engineering Department, Lehigh University, 5 East Packer Avenue, Bethlehem, PA 18015 